\title{
Limitations on the Detection Rate of High-Risk HPV by Hybrid Capture 2 Methodology in High Grade Intraepithelial (HSIL) or Atypical Squamous Cells-Cannot Exclude HSIL (ASC-H) Cytological Lesions with Proved CIN2+
}

\author{
Jean-Christophe Noël and Philippe Simon \\ Unit of Gynecopathology, Erasme University Hospital-ULB, Brussels, Belgium \\ Correspondence should be addressed to Jean-Christophe Noël; jean-christophe.noel@erasme.ulb.ac.be
}

Received 31 July 2015; Accepted 26 August 2015

Academic Editor: Ekaterina S. Jordanova

Copyright (C) 2015 J.-C. Noël and P. Simon. This is an open access article distributed under the Creative Commons Attribution License, which permits unrestricted use, distribution, and reproduction in any medium, provided the original work is properly cited.

\begin{abstract}
Recent literature data suggest that the high-risk human papillomaviruses (HR-HPVs) testing with several molecular techniques could be an alternative to cytology in the detection of cervical intraepithelial neoplasias of grade 2 or worse (CIN2+). However, any molecular techniques have its own limits and may give false negative results which must be clearly known before undertaking a primary HPV screening. This study aims to evaluate the performance of the high-risk HPV hybrid capture II detection kit (HCII) which is considered as a "gold standard technique" in a series of 100 women having proved both cytological lesions of atypical squamous cells-cannot exclude an HSIL (ASC-H) or high-grade squamous intraepithelial lesion (HSIL) and histological lesions of CIN2+. The clinical sensitivity of HCII in women with a cytological diagnosis of ASC-H/HSIL and a diagnosis of CIN2+ is high but not absolute and estimated at $96 \%$ (95,6\% and 100\% of women with a diagnosis of CIN2/3 or invasive squamous cell carcinoma, resp.). These data although they are infrequent must be clearly referred before to start an HPV primary screening of CIN2+ especially with HCII methodology.
\end{abstract}

\section{Introduction}

In Belgium, invasive cervical cancer is the 12 th most common cancer type in women and about 600 new cases are diagnosed annually [1]. In our country, as in others, "high-risk HPVs" (HR-HPVs) (HPVs: 16, 18, 31, 33, 35, 39, 45, 51, 52, 56, 58 , and 59) are clearly associated with invasive cervical cancers and preinvasive neoplastic intraepithelial lesions (CIN: cervical intraepithelial neoplasia) [2-5]. In addition to these HR-HPVs, eight other HPV types $(26,53,66,67$, 70,73 , and 82 ) have also been classified as probably or possibly carcinogenetic in recent studies [5]. The evidence of the role of HR-HPVs in the genesis of cervical cancer brought the Belgian health care experts to consider that the HR-HPV testing could be an alternative to the cytology in the detection of CIN2+ lesions in particular in women over 30 years [1]. Indeed, in agreement with several randomized studies, HR-HPVs detection is more sensitive than cytology in the detection of CIN2+ lesions and has superior efficacy to lower the incidence of invasive cervical cancer $[1,6-10]$. If this hypothesis of the screening of HR-HPVs instead of cytology in the detection of CIN2+ is attractive, it should not obscure the fact that any molecular technique for HR-HPVs detection has its own limits and may give false negative results [10]. Of course, these limits must be clearly known before undertaking a primary HPV screening but nevertheless these pieces of information are starved in the Belgian Frenchspeaking part. In order to clarify these data, we decided to analyze the percentage of HR-HPVs positivity in lesions proven both on the one hand to be cytologically highgrade squamous intraepithelial lesions ("HSIL") or atypical squamous cells-cannot exclude HSIL (ASC-H) and in the other hand to be associated with histological lesions of CIN2+. We used the Hybrid Capture 2 test (HC2, Digene 


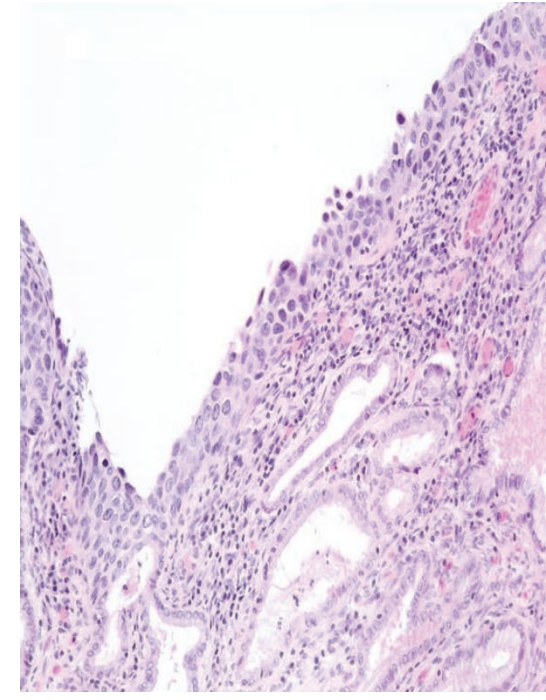

(a)

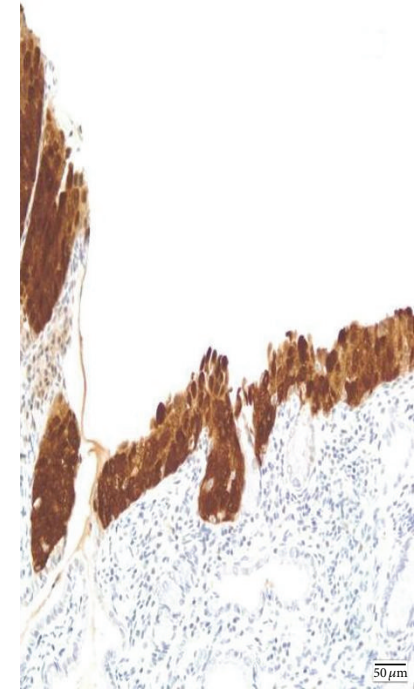

(b)

Figure 1: Typical example of CIN2/3 lesion HPV-negative (H\&E) (a). Note the strong nuclear positivity for p16 protein (b).

Corporation Gainthersburg, USA) which is considered as one of the possible molecular "standard" techniques of HR-HPV [11].

\section{Material and Methods}

2.1. Sample Collection, Cytology, and p16 Immunohistochemistry. This study was performed according to the rules of the Erasme Hospital's Ethics Committee (ethics committee approval references: P2015/234). 100 consecutive patients with a proven HSIL $(n=62)$ or ASC-H $(n=38)$ on cytology and CIN2+ lesions on biopsy (90 CIN 2/3 lesions and 10 invasive carcinomas/IC) were included in the study. All the patients (medium age: 42 years old; range: $25-77$ ) were living in either Wallonia or Brussels ("Belgian French Community"). For the cytology, as previously published, we used a thin-layer technology (ThinPrep, Cytyc Corp., Boxborough, USA) from $20 \mathrm{~mL}$ vials that contained the collected cells (PreservCyt, Cytyc Corp., Boxborough, USA) as we have previously described $[3,12,13]$. The criteria used for the cytological and histological diagnosis were those reported, respectively, by the Bethesda System for reporting cervical cytology and by the WHO classification of tumor of female reproductive organs [14-16]. In addition, to exclude mimics of CIN2/3 lesions including immature squamous metaplasia, inflammation, or atrophy, an adjunctive p16 immunohistochemistry was also applied for each biopsy as previously published and was strongly positive in all the cases (Figure 1) [16].

2.2. Hybrid Capture 2 Assay. For HPV detection we used the Hybrid Capture 2 test with RNA probe cocktail for the following HR-HPV types 16, 18, 31, 33, 35, 39, 45, 51, 52, 56, 58,59 , and 68 from a residual quantity of $4 \mathrm{~mL}$ of PreservCyt vial after cytology as we have previously described $[12,13,17]$. In this study, the HC2 test was interpreted as positive when the ratio of relative light units (RLU/CO) to the positive control specimen was $>1.0$.

\section{Results}

96 of the 100 (96\%) of HSIL/ASC-H cytological lesions with a CIN2+ lesion on biopsy were HR-HPV-positive with HC2. From them, 10 cases with a histological diagnosis of invasive carcinoma (ISCC) were positive (100\%). In ASC-H or HSIL $(n=90)$ with CIN2/3 lesions on biopsy a positivity was observed in 86 cases (95,6\%). The results are summarized in Table 1 .

\section{Discussion}

The fact that firstly HR-HPV infection is a necessary cause for the development of CIN2+ lesions and secondly that DNA HPV testing has a higher sensitivity and reproducibility than cytology has led several countries including Belgium to the fact that a switch from cytology to HR-HPV detection could constitute an interesting alternative for cervical cancer screening [1, 2, 6-10]. In Belgium, it has been proposed that a technique to be validated for the primary screening should have a sensitivity at least equivalent to that from the GP5+/6+ PCR-based enzyme immunoassay (PCR EIA) (EIA: Diassay, Rijswijk, Netherlands) or HCII assay [1]. Indeed, these two tests have been extensively clinically validated particularly in the triage of borderline cytological lesion for colposcopy and have been considered, perhaps erroneously, as "references gold standard" [11]. However, in our country, at least in its French part, we do not have clear data regarding potential false negatives that might be caused by these techniques if they were used as a primary screening. In preliminary studies, particularly optimistic, it was suggested that the sensitivity of HCII assay for detecting histologically confirmed highgrade squamous intraepithelial lesions (CIN 2/3) is 100\% [18]. 
TABLE 1: Correlations between cytological and histological diagnosis and HR-HPVs positivity with HCII methodology.

\begin{tabular}{lcc}
\hline Cytological diagnosis & Histological diagnosis & HR-HPVs positivity with HCII methodology \\
\hline ASC-H $(n=32)$ & CIN2/3 & $31(97 \%)$ \\
ASC-H $(n=6)$ & ISCC & $6(100 \%)$ \\
HSIL $(n=58)$ & CIN2/3 & $55(95 \%)$ \\
HSIL $(n=4)$ & ISCC & $4(100 \%)$ \\
\hline ASC-H or HSIL $(\mathbf{n}=\mathbf{1 0 0})$ & CIN2+ & $\mathbf{9 6 ( 9 6 \% )}$ \\
\hline
\end{tabular}

In our work, we do not share this view because we have clearly demonstrated that at least $4 \%$ of women with highly atypical cytological lesions (ASC-H or HSIL) with CIN2+ diffusely immunoreactive for p16 protein are clearly negative by HCII test. Despite the fact that the sensitivity and specificity of p16 are not perfect, our results are consistent with recent literature data confirming that the sensitivity of the most widely validated tests for use in primary screening in the detection of CIN2+ lesions such as HCII test, GP5+/6+ PCR EIA assay, Cobas 4800 PCR assay (Roche molecular systems, Inc., Alameda, CA, USA), or Abbott real-time high-risk HPV PCR (Abbott Molecular, Des Plaines, IL, USA) is variable ranging from 95 to $97 \%$ (for the more optimistic studies and so forth) [11, 19-21]. The cause of the lack of "absolute" sensitivity in the detection of CIN2+ lesions is probably multiple and includes the sensitivity of the technique itself, the possibility that the regression of HPV infection may precede the regression of histological lesions or simply that the HPV causing CIN2+ lesions is not present in the various HPV cocktail provided in the different tests $[5,11]$. However, it is interesting to note that, in this study, all cases of ISCC with a positive cytology were detected by HCII methodology, which is reassuring information altogether. Obviously, our study has limitations, firstly because the CIN2 or 3 lesions have not been differentiated and secondly because mainly not only CIN2 but also CIN3 may regress.

In conclusion, HPV primary screening has a significantly better sensitivity (>90\% even $95 \%$ ) than cytology and appears as a future robust methodology for a large and rapid screening. Nevertheless, contrary to a conventional wisdom, we demonstrated that, in our population, there were highgrade or suggested high-grade cytological abnormalities with clearly proven CIN2+ lesions for which the research of viral HR HPVs sequences with HCII methodology was negative. These data, although they are infrequent, must be known and referred before to start an HPV primary screening of CIN2+ lesions especially with HCII methodology which is considered by many as a reference standard technology particularly in Belgium.

\section{Conflict of Interests}

Jean-Christophe Noël and Philippe Simon have disclosed no potential conflict of interests.

\section{References}

[1] M. Arbyn, A. Haelens, A. Desomer et al., "Cervical cancer screening program and Human Papillomavirus (HPV) testing: update on HPV primary screening," KCE Reports 238, Health Technology Assessment (HTA), Belgian Health Care Knowledge (KCE), Brussels, Belgium, 2015.

[2] A. R. Giuliano, A. G. Nyitray, A. R. Kreimer et al., "EUROGIN 2014 roadmap: differences in human papillomavirus infection natural history, transmission and human papillomavirusrelated cancer incidence by gender and anatomic site of infection," International Journal of Cancer, vol. 136, no. 12, pp. 27522760, 2015.

[3] X. Catteau, P. Simon, and J. Noël, "Evaluation of the oncogenic human papillomavirus DNA test with liquid-based cytology in primary cervical cancer screening and the importance of the ASC/SIL ratio: a belgian study," ISRN Obstetrics and Gynecology, vol. 2014, Article ID 536495, 5 pages, 2014.

[4] F. Coutlée, S. Ratnam, A. V. Ramanakumar et al., "Distribution of human papillomavirus genotypes in cervical intraepithelial neoplasia and invasive cervical cancer in Canada," Journal of Medical Virology, vol. 83, no. 6, pp. 1034-1041, 2011.

[5] G. Halec, L. Alemany, B. Lloveras et al., "Pathogenic role of the eight probably/possibly carcinogenic HPV types 26, 53, 66, 67, 68, 70, 73 and 82 in cervical cancer," The Journal of Pathology, vol. 234, no. 4, pp. 441-451, 2014.

[6] D. C. Rijkaart, J. Berkhof, L. Rozendaal et al., "Human papillomavirus testing for the detection of high-grade cervical intraepithelial neoplasia and cancer: final results of the POBASCAM randomised controlled trial," The Lancet Oncology, vol. 13, no. 1, pp. 78-88, 2012.

[7] M. Leinonen, P. Nieminen, L. Kotaniemi-Talonen et al., "Agespecific evaluation of primary Human Papillomavirus screening vs conventional cytology in a randomized setting," Journal of the National Cancer Institute, vol. 101, no. 23, pp. 1612-1623, 2009.

[8] G. Ronco, P. Giorgi-Rossi, F. Carozzi et al., "Efficacy of human papillomavirus testing for the detection of invasive cervical cancers and cervical intraepithelial neoplasia: a randomised controlled trial," The Lancet Oncology, vol. 11, no. 3, pp. 249-257, 2010.

[9] J. Cuzick, C. Clavel, K.-U. Petry et al., "Overview of the European and North American studies on HPV testing in primary cervical cancer screening," International Journal of Cancer, vol. 119, no. 5, pp. 1095-1101, 2006.

[10] P. E. Castle, S. de Sanjose, Y.-L. Quiao, J. L. Belinson, E. LazcanoPonce, and W. Kinney, "Introduction of human papillomavirus DNA screening in the world: 15 years of experience," Vaccine, vol. 30, supplement 5, pp. F117-F122, 2012.

[11] J. S. Gibson, "Nucleic acid-based assays for the detection of high-risk human papillomavirus: a technical review," Cancer Cytopathology, vol. 122, no. 9, pp. 639-645, 2014.

[12] X. Catteau, M. Vanhaeverbeek, and J.-C. Noël, "Importance of the residual volume of the cytological solution for the reproducibility of the hybrid capture 2 high-risk human papillomavirus DNA test," Acta Cytologica, vol. 56, no. 4, pp. 375-378, 2012. 
[13] J.-C. Noël, I. Fayt, M. R. Romero Munoz, P. Simon, and C. Engohan-Aloghe, "High prevalence of high-risk human papillomavirus infection among women with Trichomonas vaginalis infection on monolayer cytology," Archives of Gynecology and Obstetrics, vol. 282, no. 5, pp. 503-505, 2010.

[14] F. W. Abdul-Karim, C. N. Powers, J. S. Berek et al., "A typical squamous cells," in The Bethesda System for Reporting Cervical Cytology, R. Nayar and D. C. Wilbur, Eds., pp. 103-134, 3rd edition, 2015.

[15] M. R. Henry, D. K. Russell, R. D. Luff et al., "Epithelial cell abnormalities: squamous," in The Bethesda System for Reporting Cervical Cytology, R. Nayar and D. C. Wilbur, Eds., pp. 135-192, 3rd edition, 2015.

[16] M. Stoler, C. Bergeron, T. J. Colgan et al., "Squamous cell tumours and precursors," in WHO Classification of Tumours of Female Reproductive Organs, R. J. Kurman, M. L. Carcangiu, C. S. Herrington, and R. H. Young, Eds., pp. 172-198, IARC, Lyon, France, 4th edition, 2014.

[17] X. Catteau, P. Simon, and J.-C. Noël, "Detection of high-grade lesions on cell blocks from residual fluids of pap smears diagnosed as low-grade abnormalities: a preliminary pilot study," Acta Cytologica, vol. 56, no. 3, pp. 247-250, 2012.

[18] C. Clavel, M. Masure, J.-P. Bory et al., "Human papillomavirus testing in primary screening for the detection of high-grade cervical lesions: a study of 7932 women," British Journal of Cancer, vol. 89, no. 12, pp. 1616-1623, 2001.

[19] A. Szarewski, D. Mesher, L. Cadman et al., "Comparison of seven tests for high-grade cervical intraepithelial neoplasia in women with abnormal smears: the predictors 2 study," Journal of Clinical Microbiology, vol. 50, no. 6, pp. 1867-1873, 2012.

[20] J. Cuzick, L. Cadman, D. Mesher et al., "Comparing the performance of six human papillomavirus tests in a screening population," British Journal of Cancer, vol. 108, no. 4, pp. 908913, 2013.

[21] J. Dillner, M. Rebolj, P. Birembaut et al., "Long term predictive values of cytology and human papillomavirus testing in cervical cancer screening: joint European cohort study," British Medical Journal, vol. 377, Article ID a1754, 2008. 


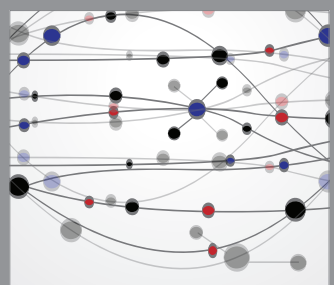

The Scientific World Journal
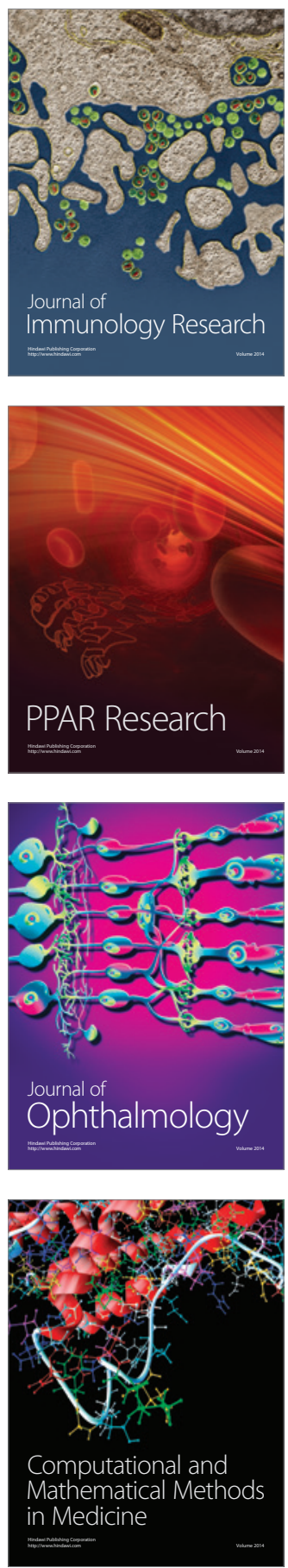

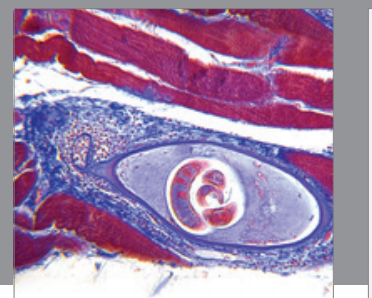

Gastroenterology

Research and Practice
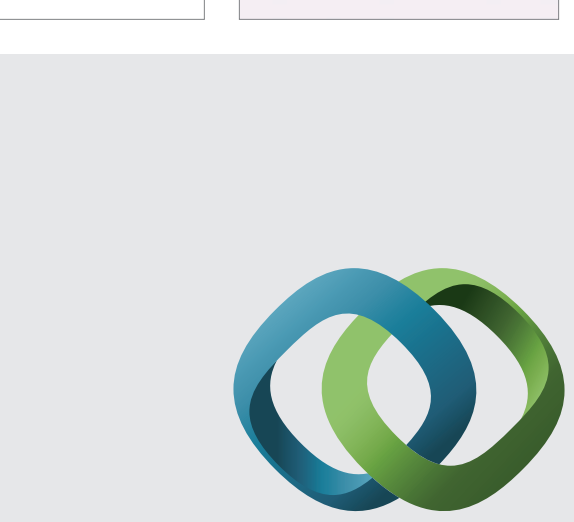

\section{Hindawi}

Submit your manuscripts at

http://www.hindawi.com
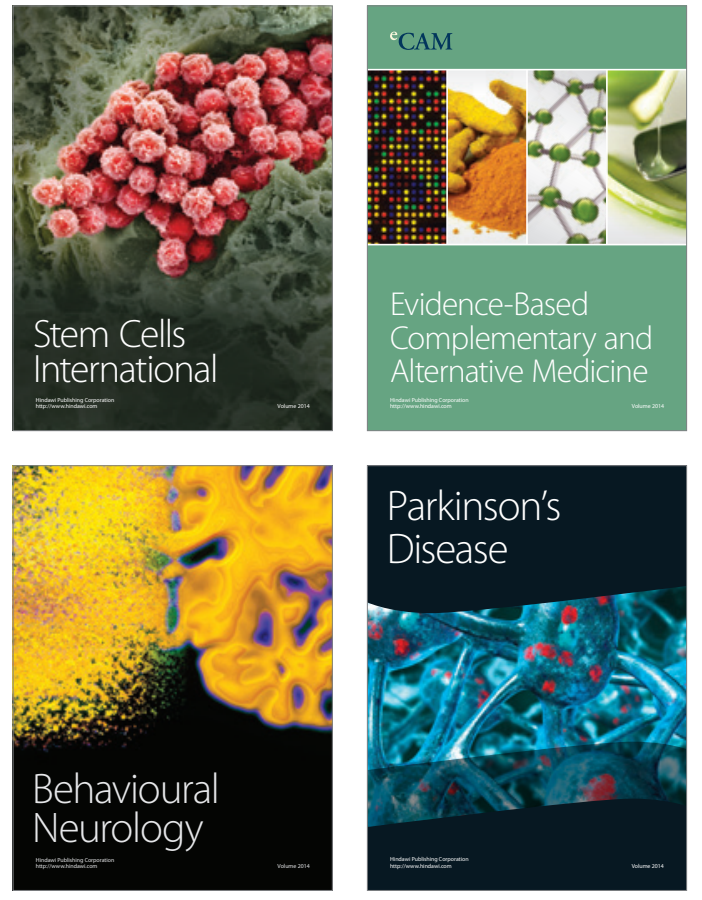
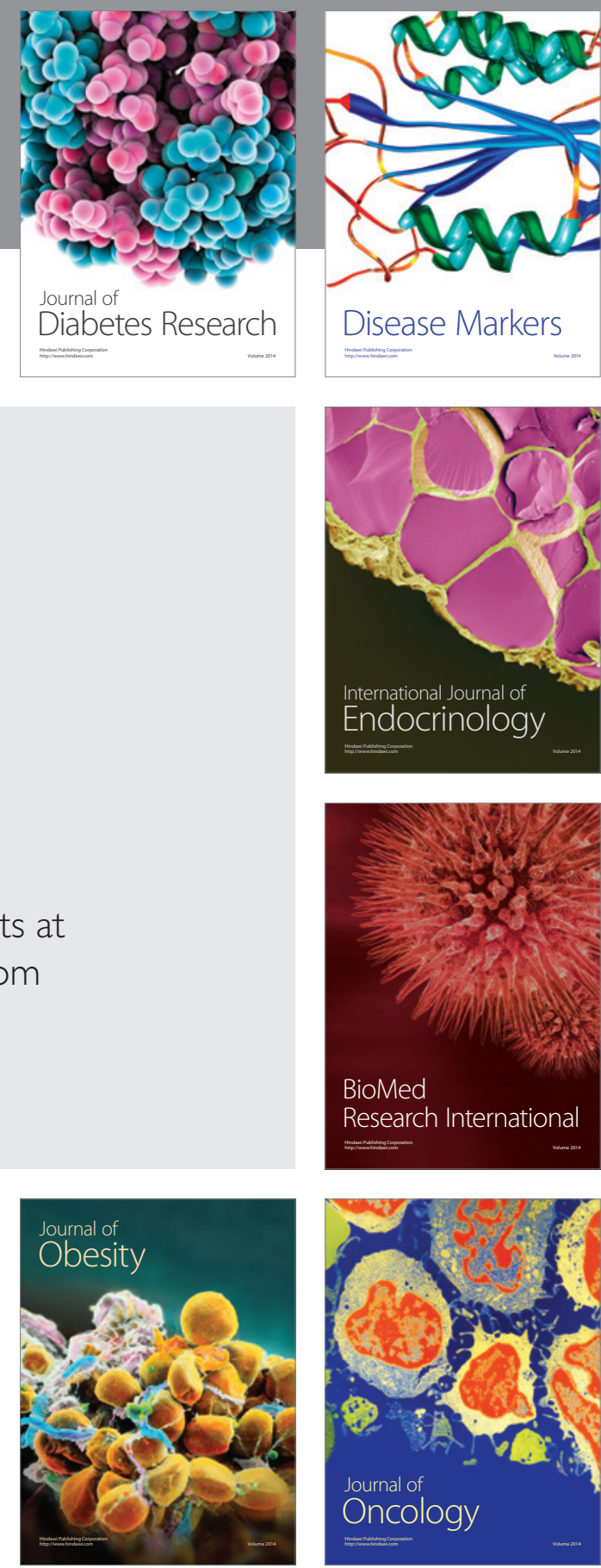

Disease Markers
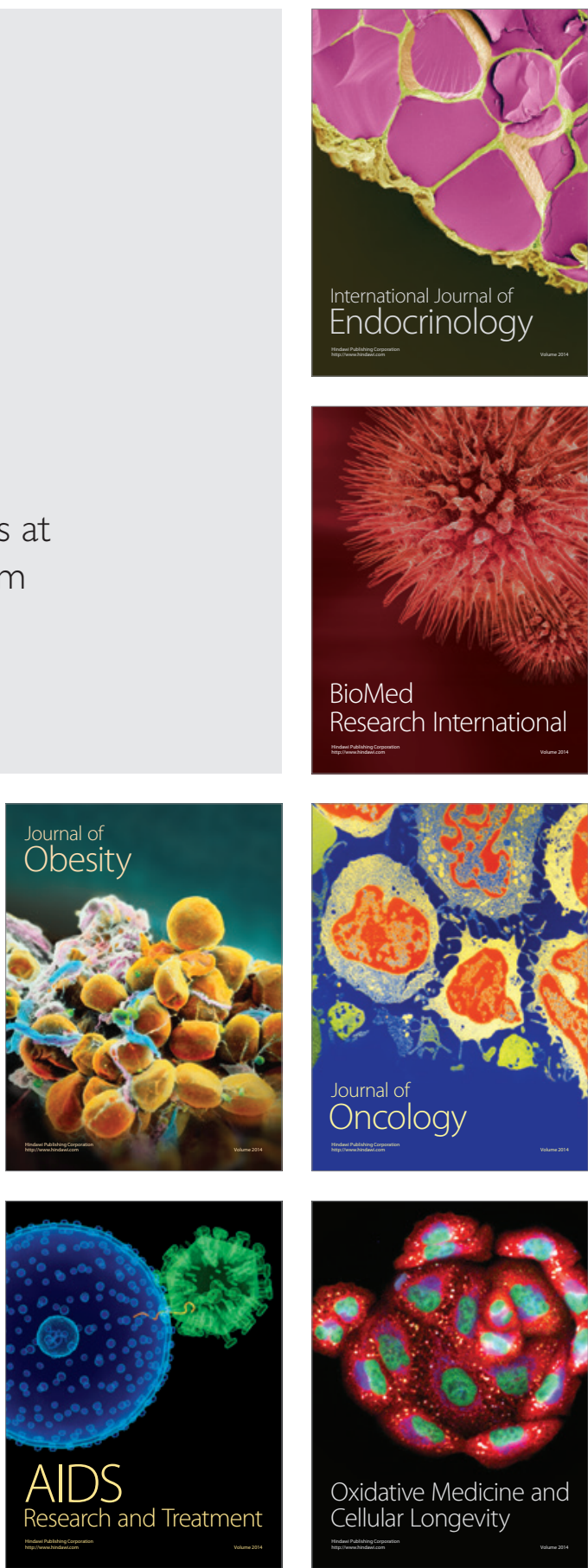